\title{
Impact of Traffic Sign on Pedestrians' Walking Behavior
}

\author{
Hui Xiong, ${ }^{1}$ Pingfu Yao, ${ }^{1}$ Xuedong Guo, ${ }^{1}$ Chenglong Chu, ${ }^{2}$ and Wuhong Wang ${ }^{1}$ \\ ${ }^{1}$ Department of Transportation Engineering, Beijing Institute of Technology, Beijing 100081, China \\ ${ }^{2}$ Department of Construction Management, Tsinghua University, Beijing 100084, China \\ Correspondence should be addressed to Hui Xiong; xionghui@bit.edu.cn
}

Received 30 July 2014; Revised 26 October 2014; Accepted 29 October 2014

Academic Editor: Heiner Bubb

Copyright (C) 2015 Hui Xiong et al. This is an open access article distributed under the Creative Commons Attribution License, which permits unrestricted use, distribution, and reproduction in any medium, provided the original work is properly cited.

\begin{abstract}
To study the impact of traffic sign on pedestrian walking behavior, the paper applies cellular automaton to simulate one-way pedestrian flow. The channel is defined as a rectangle with one open entrance and two exits of equal width. Traffic sign showing that exit is placed with some distance in the middle front of the two exits. In the simulation, walking environment is set with various input density, width of exit, width and length of the channel, and distance of the traffic sign to exit. Simulation results indicate that there exists a critical distance from the traffic sign to exit for a given channel layout. At the critical distance, pedestrian flow fluctuates. Below such critical distance, flow is getting larger with the increase of input density. However, the flow drops sharply when the input density is over a critical level. If the distance is a little bit further than the critical distance, the largest flow occurs and the flow can remain steady no matter what input density will be.
\end{abstract}

\section{Introduction}

Pedestrian's walking behavior is much more complicated compared with the vehicle traffic due to the fact that there is no designated trail to constrain pedestrians' moving space. However, we still need to understand and predict such behavior very well to conduct sound evacuation management in emergency or to keep pedestrian facility in good operation.

In the past several decades, modeling pedestrian flow has attracted considerable attention and numerous models were proposed. Normally, pedestrian models can be in a macroscopic nature or microscopic nature. Macroscopic models are often in the form of partial differential equations. Instead of describing individual pedestrian's behavior, this type of models treats crowd as a whole and applies the conservation law to capture the relationship among speed, flow, and density of pedestrian flow. Applying conservation law, Hughes [1] derived partial differential equations for flows with single or multiple pedestrian types. How to solve Hughes' model has been attracted by many researchers thereafter [2, 3]. Colombo and Rosini [4] proposed another type of partial differential equation model for pedestrian flow with a new parameter called characteristic density that revealing the maximal density in panic. Jiang et al. [5] developed an extended reactive dynamic user equilibrium model for pedestrian counter flow. Some phenomena such as lane formation in crowd were observed in numerical simulation. Another model proposed by Jiang et al. is a twodimension high-order macroscopic model derived from fluid dynamics. Simulation results showed capability of the model to describe complex phenomena such as stop-and-go waves [6]. Henderson [7] took pedestrian in crowd as gas molecules and applied the Maxwell-Boltzmann theory to describe the velocity distribution of people movements. Without using the conservation assumptions in Henderson's study, Helbing [8] developed a fluid dynamic model for the collective movement of pedestrians based on the Boltzmann-like approach.

Microscopic pedestrian model can be described in detail as the interactions among pedestrians, or between pedestrians and obstacles. Those models include, among others, cellular automaton (CA) model [9-16], lattice gas model [1721], social force model [22], centrifugal force model [23, $24]$, floor field model $[25,26]$, and other rule-based models [27-32]. In CA models, the walking space is set as a twodimensional system and divided into cells. Each cell can either be empty, occupied by exactly one pedestrian, or occupied by wall or obstacle. For each time-step, pedestrian can move to adjacent cells or stand still complying with the route 


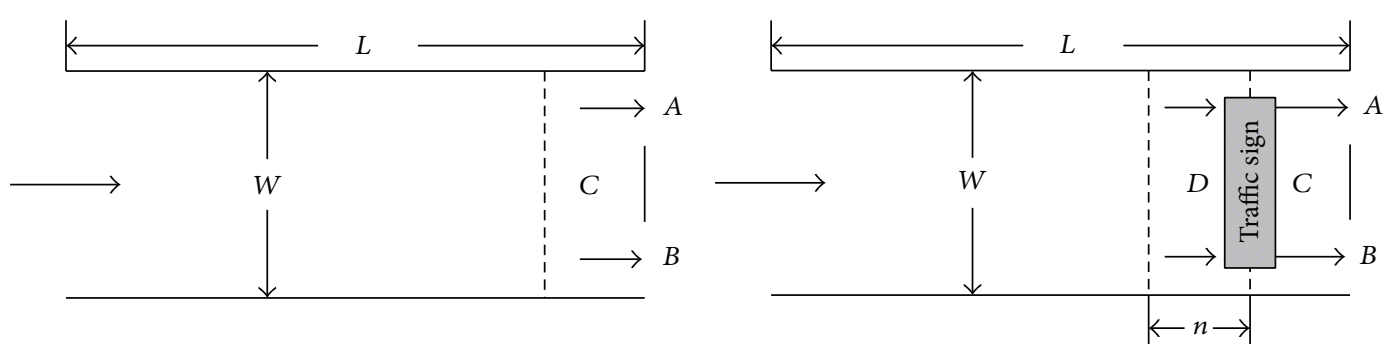

(a)

(b)

FIGURE 1: Schematic illustration of the channel with/without traffic sign.

choice rule and conflicting with the avoidance rule. In recent years, CA models are widely used for capturing pedestrian walking behaviors, such as bidirection movement $[9,10]$, counter flow with paired pedestrians [11], movement with right-moving preference $[12,13]$, counter flow with different velocities [14], impact of surrounding environment [15], and teaming-moving impact [16]. Nagatani [9] presented a bidirectional CA model for facing pedestrian flow on a wide passage. It was found that the dynamical phase transitions occurred at three stages with increasing density. Blue and Adler [10] simulated three bidirectional pedestrian flows, including flows in separated lanes, interspersed flow, and dynamic multilane flow. Results showed that the emergent fundamental parameters of speed, flow, and density produced from the model were reasonable compared with field data. Xiong et al. [11] simulated walking behavior with paired pedestrians. Considering right-hand side walking preference, Fang et al. [12] studied bidirectional pedestrian movement. Results indicated that the critical density when phase transition occurred increased as the probability of backstepping rose, and the critical density declined with the increase of the system size. Yang et al. [13] concluded that rightpreference was effective when the density was below critical value. Weng et al. [14] simulated pedestrian counterflow with different walk velocities through updates at different time-step intervals. Simulation results revealed three phases of pedestrian patterns, including freely moving phase, lane formation phase, and perfectly stopped phase. Considering the impact of surrounding environment on pedestrian flow, Yu and Song [15] introduced interaction radius parameter to route choice rules. It concluded that the critical entrance density at the transition point did not depend on the system size when the radius was large. Wang et al. [16] revealed that team-moving could significantly influence corridor capacity by means of teaming manner.

Pedestrian's walking behavior is affected by many factors including walker's characteristic and walking channel environment. Among these factors, traffic sign is a key impact factor to those who are not familiar with the walking environment. A case study showed that pedestrian walking behavior was affected by content and position of the sign [33]. According to a questionnaire survey we conducted, as high as $97.4 \%$ (74 of 76 ) subway riders reported that traffic sign showing exit were helpful and they would change their moving direction accordingly in an unfamiliar station. Liu et al. [34] proposed a computational model for determination of the effective distance of emergency evacuation signs. Nassar [35] proposed an optimization framework for signs location to increase their detectability in public space. The aim of this paper is to study the impact of traffic sign on pedestrian behavior by means of CA model.

The paper is structured as follows. Section 2 develops the cellular automaton model and defines walking rules for pedestrians with traffic sign in system. Section 3 applies the model to simulate pedestrian movement in various scenarios. In this section, some meaningful phenomena relating to the function of traffic sign are studied. The conclusions are presented in Section 4.

\section{Model}

We apply a two-dimensional cellular automaton to define the walking channel. The underlying structure is a $W \times L$ cell grid, where $W$ is the width of the channel and $L$ is the length of the channel. The entrance is at the left with width of $W$, and two exits, denoted by $A$ and $B$, are at the right boundary with equal width of $w$. Each cell can either be empty or occupied by wall, obstacle, or exactly one pedestrian. The size of a cell is set to be $0.4 \times 0.4 \mathrm{~m}^{2}$. This is a typical space occupied by a person in a dense crowd [28]. Each pedestrian can move only one cell per time-step. In practice, the average velocity of a pedestrian is about $1.0 \mathrm{~ms}^{-1}$ under normal circumstances [29]. So the time-step is approximately $0.4 \mathrm{~s}$.

The channel is illustrated in Figure 1. In Figure 1(a), there is no traffic sign and pedestrians can recognize exits only when they crossed the dashed line in area $C$. In Figure 1(b), we set traffic sign in the middle front of the two exits. Pedestrians who are in area $D$ can see the traffic sign pointing at the proper exit.

Two types of walkers are taken into account: walkers who take exit $A$ and walkers who take exit $B$. The numbers of the two types of walkers entering from the left entrance are equal. The channel is confined with walls at top and bottom boundaries. Anyone can walk only from left to right, but no one can cross the top or bottom boundaries.

Taking pedestrians who are choosing exit $A$ for example, there are three typical positions for each pedestrian; see Figure 2. With traffic sign, pedestrian $a$ and pedestrian $b$ are in the area with impact of traffic sign, whereas pedestrian $c$ is out of such area. In this case, $a$ and $c$ keep walking 


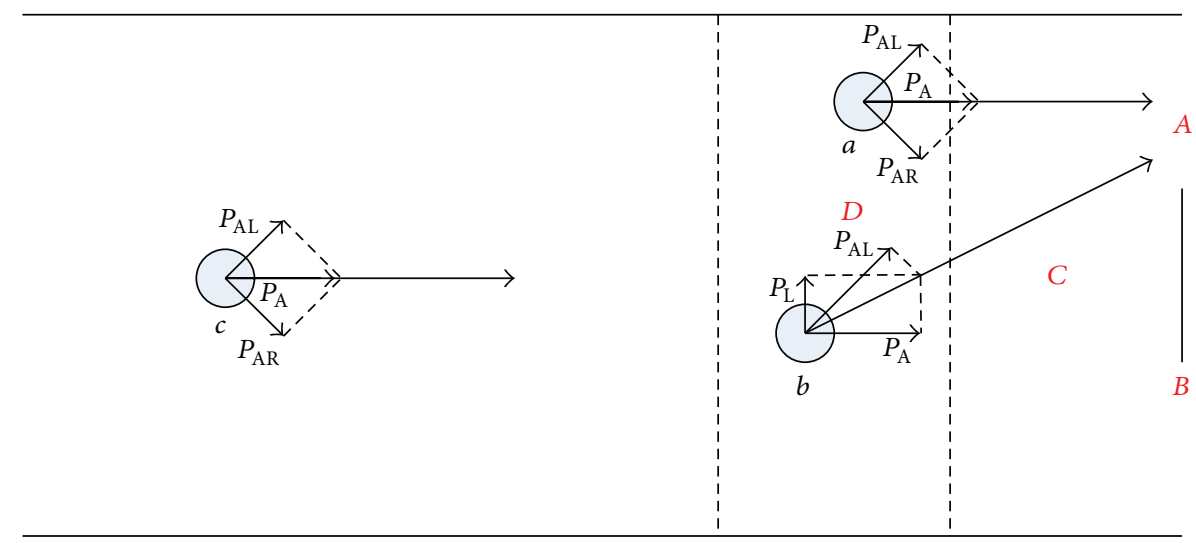

FIGURE 2: Pedestrian's moving direction without obstacles.

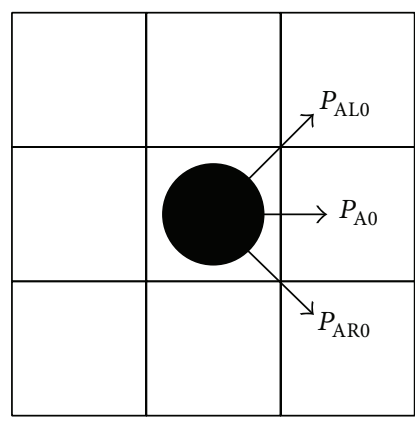

(a)

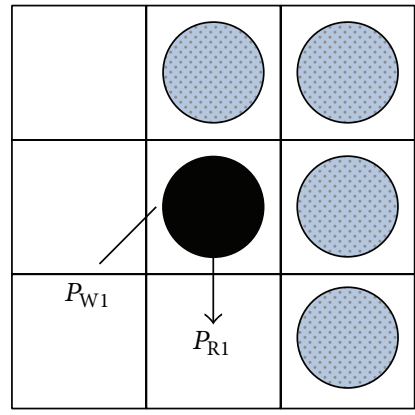

(d)

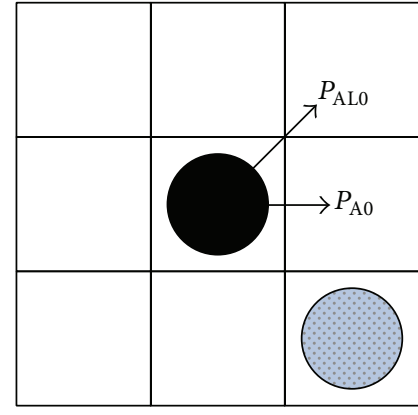

(b)

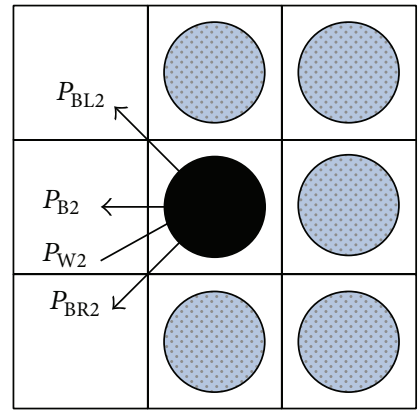

(e)

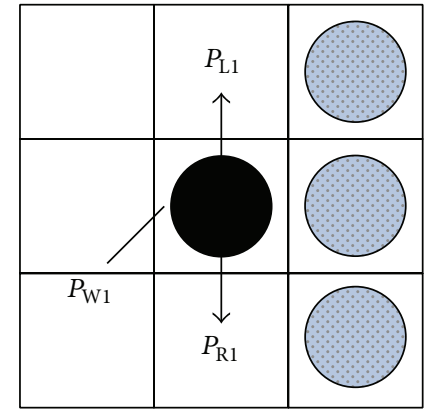

(c)

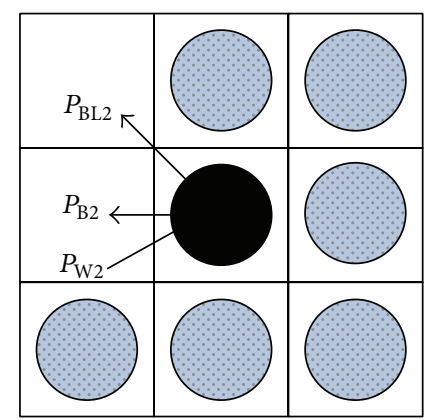

(f)

FIGURE 3: Some typical configurations that pedestrians $a$ and $c$ may encounter.

forward and $b$ will adjust its direction to get close to exit $A$. Probability of a pedestrian moving forward is denoted by $P_{A}$, and probabilities of moving left forward, right forward, and left are denoted by $P_{A L}, P_{A R}$, and $P_{L}$, respectively.

The update is random sequential for all pedestrians at every time-step. Usually a parallel update rule is considered to be more realistic, but it might lead to conflicts in the update [36]. Figure 3 shows all the possible configurations that pedestrians $a$ and $c$ may encounter. If the front adjacent cells are not totally occupied, the probabilities to move forward, left forward, and right forward are determined by the angle between moving direction and the horizontal line. In Figure 3(a), if all front cells are empty, the walker will select the one of the three front cells with probability denoted by
$P_{A 0}, P_{A L 0}$, and $P_{A R 0}$, where $P_{A 0}, P_{A L 0}$, and $P_{A R 0}$ are expressed as $\eta \cos \theta_{i j}, \eta$ is normalization parameter to assure $P_{A 0}+P_{A L 0}+$ $P_{A R 0}=1$, and $\theta_{i j}$ is angle between moving direction and the horizontal line. In Figure 3(b), if both the upper cell and the front cell are free, the walker will select any of the two cells to move in with probability $P_{A 0}$ or $P_{A L 0}$. For configuration (c), the probability of selecting the left, right adjacent cell or waiting is denoted by $P_{L 1}, P_{R 1}$, and $P_{W 1}$, respectively, where $P_{L 1}=P_{R 1}=P_{W 1}=1 / 3$. For configuration (d), the probability of selecting the right adjacent cell or waiting is $P_{R 1}$ and $P_{W 1}$, respectively, where $P_{R 1}=P_{W 1}=1 / 2$. For configuration (e), the probability of selecting the left rear, right rear, back adjacent cell, and waiting is denoted by $P_{B L 2}, P_{B R 2}, P_{B 2}$, and $P_{W 2}$, respectively, where $P_{B L 2}=P_{B R 2}=P_{B 2}=P_{W 2}=1 / 4$. For 


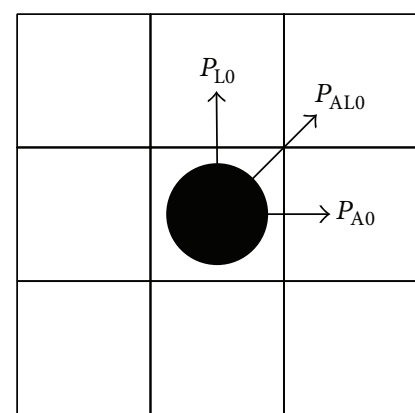

(a)

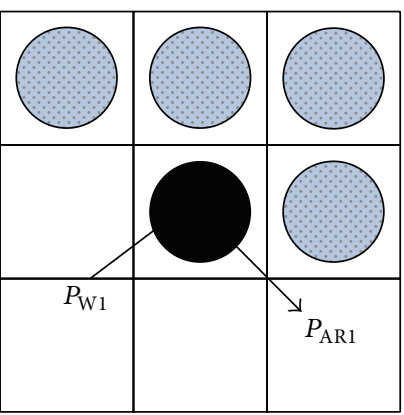

(d)

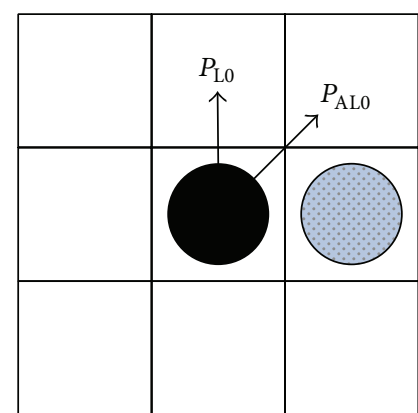

(b)

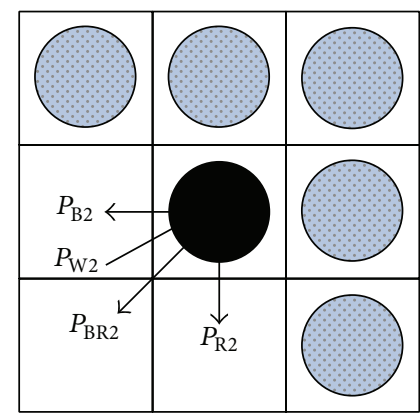

(e)

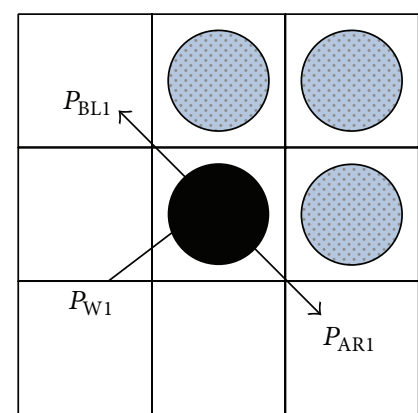

(c)

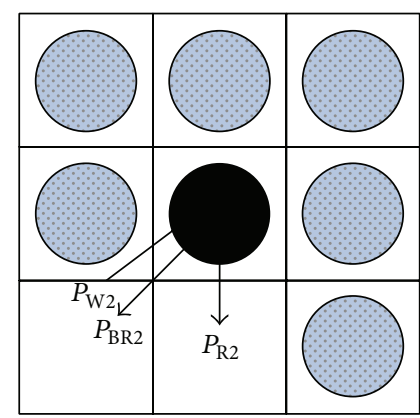

(f)

FIGURE 4: Some typical configurations that pedestrian $b$ may encounter.

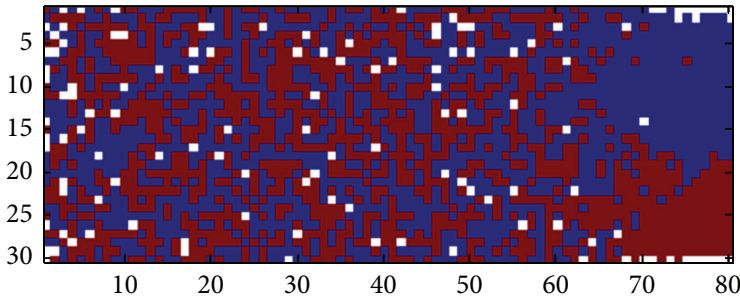

(a)

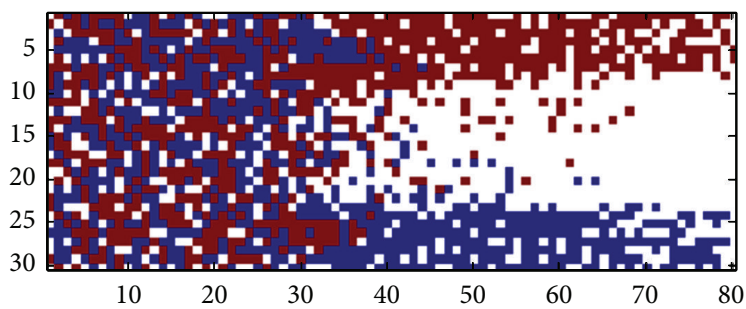

(c)

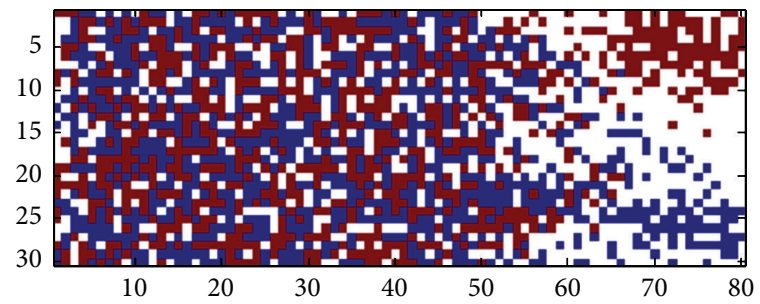

(b)

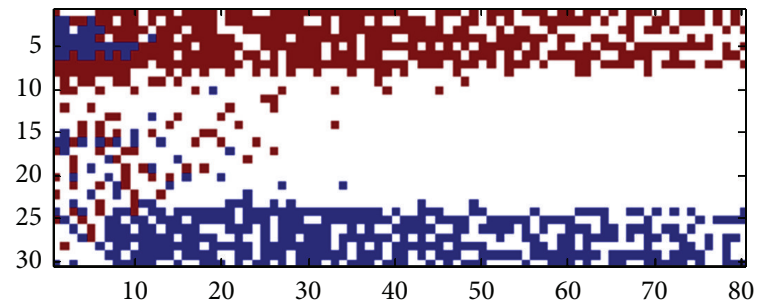

(d)

FIGURE 5: Pedestrians' moving state in the channel with different input density. The length of channel is 80 and the width is 30 . Input density is set as 0.5 . Impact distance is set as 10, 30, 50, and 80 cells corresponding to (a), (b), (c), and (d), respectively. The cell in red represents pedestrian who will take exit $A$ and the cell in blue represents pedestrian who will take exit $B$.

configuration (f), the probability of selecting the left rear or back adjacent cell or waiting is denoted by $P_{B L 2}, P_{B 2}$, and $P_{W 2}$, respectively, where $P_{B L 2}=P_{B 2}=P_{W 2}=1 / 3$.

Figure 4 shows all the possible configurations that pedestrian $b$ may encounter. In Figure 4(a), if all front cells are empty, the walker will select the one of the three front cells with probability denoted by $P_{A 0}, P_{A L 0}$, and $P_{A R 0}$, where $P_{A 0}$,
$P_{A L 0}$, and $P_{A R 0}$ are expressed as $\eta \cos \theta_{i j}, \eta$ is normalization parameter to assure $P_{A 0}+P_{A L 0}+P_{A R 0}=1$, and $\theta_{i j}$ is angle between moving direction and the horizontal line. In Figure 4(b), if the front cell is occupied, the walker will select the left or left front cell to move in with probability $P_{A 0}$ or $P_{A L 0}$. For configuration (c), the probability of selecting the left rear, right front adjacent cell, or waiting is $P_{B L 1}, P_{A R 1}$, and 


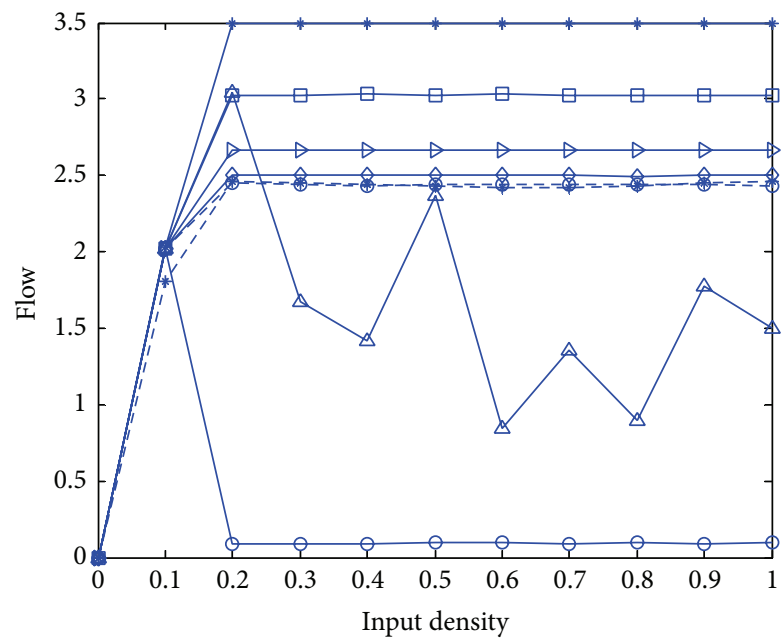

(a)

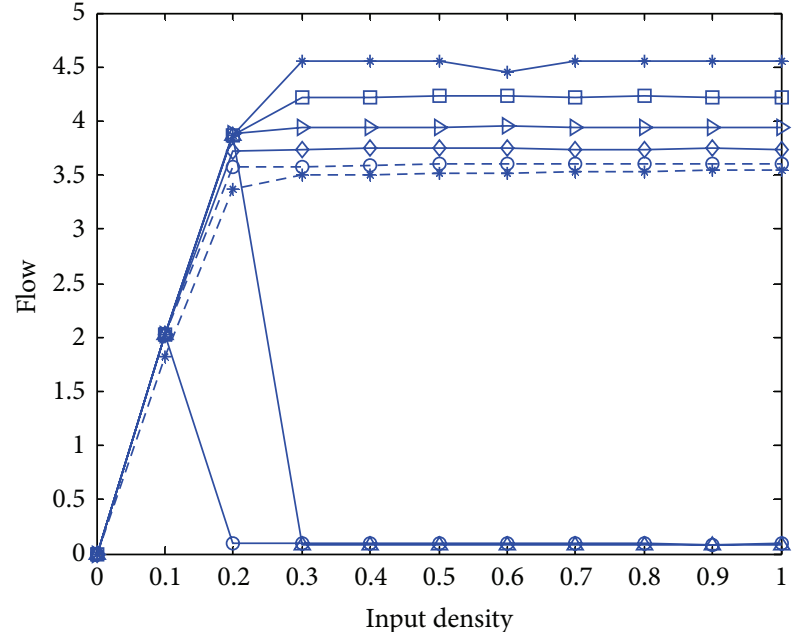

(b)

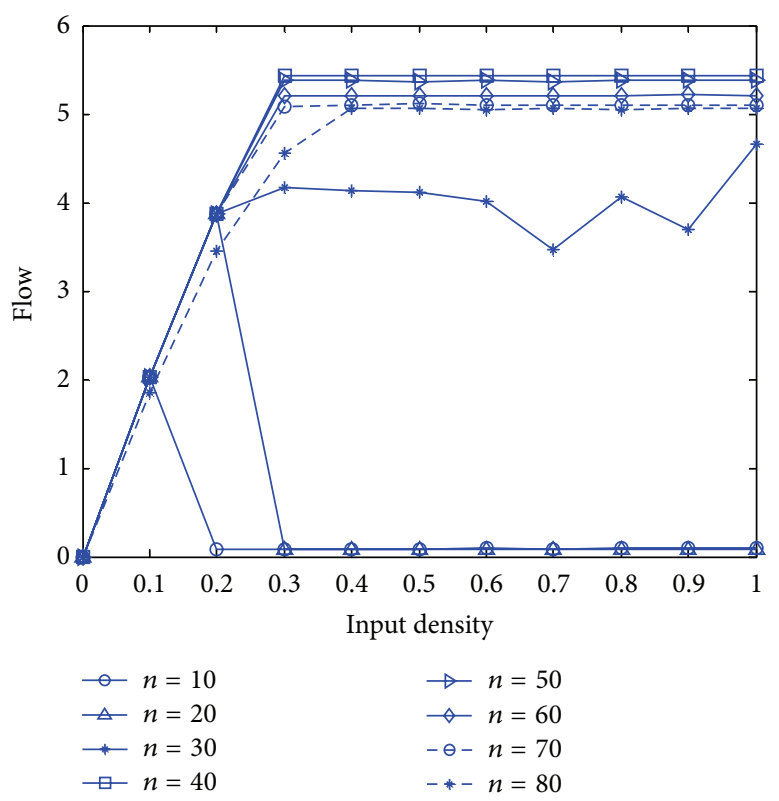

(c)

Figure 6: Relationship between flow and input density at different impact distance. The length of channel is 80 and the width is 30 . The width of exit is set as 5, 7, and 10 cells corresponding to (a), (b), and (c), respectively.

$P_{W 1}$, respectively, where $P_{B L 1}=P_{A R 1}=P_{W 1}=1 / 3$. For configuration $(\mathrm{d})$, the probability of selecting the right front adjacent cell or waiting is $P_{A R 1}$ and $P_{W 1}$, respectively, where $P_{A R 1}=P_{W 1}=1 / 2$. For configuration (e), the probability of selecting the rear, right rear, right adjacent cell, or waiting is $P_{B 2}, P_{B R 2}, P_{R 2}$, and $P_{W 2}$, respectively, where $P_{B 2}=P_{B R 2}=$ $P_{R 2}=P_{W 2}=1 / 4$. For configuration (f), the probability of selecting the right rear, right adjacent cell, or waiting is $P_{B R 2}$, $P_{R 2}$, and $P_{W 2}$, respectively, where $P_{B R 2}=P_{R 2}=P_{W 2}=1 / 3$.

\section{Simulation and Results}

To study the impact of traffic sign on pedestrian walking behavior, we analyze changes of pedestrian flow with various input density, impact area of the sign, and channel configuration. Pedestrian flow is defined as the number of pedestrians crossing the right boundary at each time-step. Input density is defined as the number of entering pedestrians at each timestep over the width of the channel. Input density is set as a constant in each scenario. Impact distance is defined as the furthest distance for a pedestrian to recognize the traffic sign, denoted as $n$ in Figure 1(b). At the very beginning, the channel is empty. For each simulation, total 150,000 time-steps are carried out, and the value of averaged flow is calculated according to the results of the last 5,000 time-steps. We take the average of the 10 iteration results as the final value.

Pedestrians are assumed to recognize the sign as soon as they stepped into the impact area. Simultaneously, they 


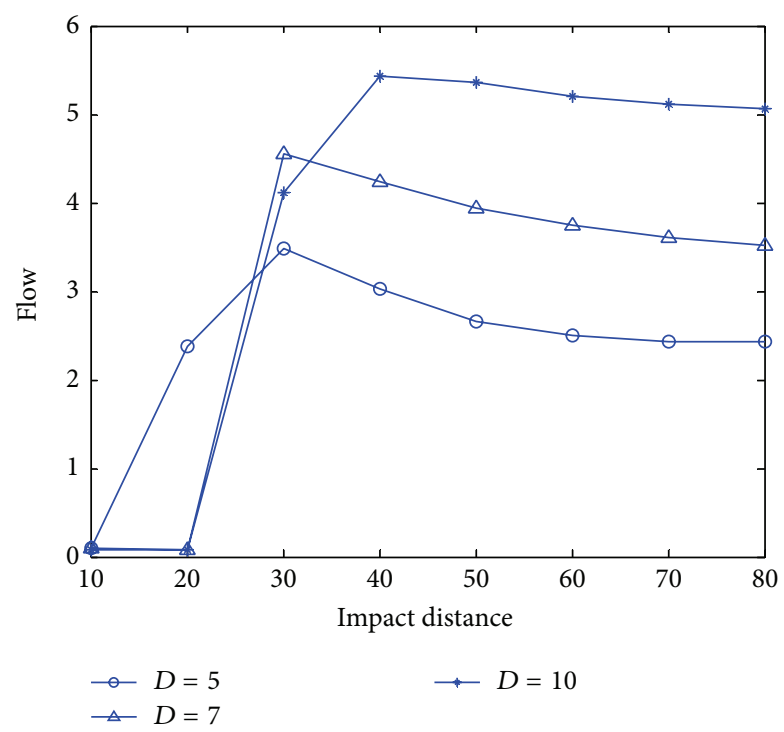

FIgURE 7: Relationship between flow and impact distance of traffic sign. The length of channel is 80 and the width is 30 . The width of exit is set as 5,7 , or 10 , respectively.

started to adjust moving direction towards the preferred exit. Kretz et al. [37] analyzed such psychological act in a tunnel fire simulation. For the sake of simplicity, we ignored decision process from cognition to perception. In simulation, the height of a pedestrian is not considered, which means the front pedestrian will never block the sight of the follower.

Figure 5 shows pedestrians' moving state with impact of traffic sign at different distances. It shows that, with impact distance of 10 cells, pedestrians are blocked in front of the exits. Those who are aiming at taking exit $B$ are blocked in front of exit $A$ and vice versa. In this case, no one can get out of the channel. If we set impact distance at a further distance, say 30 cells, pedestrian can recognize his/her exit in advance and adjust the moving direction accordingly within the space to the exit. The further we set the traffic sign to the exits, the earlier pedestrians recognize the proper exit, and the easier it is to move out of the channel.

In Figure 6 we show relationship between flow and input density with different exit width or different impact distance. If we set the impact distance as 10 cells and input density as 0.1 , pedestrian flow will be about 2.0 no matter what width of the exit we set. But with input density of 0.2 or larger, the flow decreases dramatically to 0 . On the other hand, if we set impact distance further and further, we can see that with the same input density, flow is getting lower and lower. It indicates that, in real world, traffic sign should be set at an appropriate distance to exit. It is of no use to set traffic sign too far or too close to the exits.

We can see the fluctuation of flow versus input density. In reality, flow is influenced by input density, width of the channel and exits, location of the sign, and so on. Pedestrians tried to change moving direction when they noticed the sign. In some circumstances, pedestrians blocked each other.
Such situation is well described by the asymmetric simple exclusion process (ASEP) [38-41].

To study impact of the exit width on the relationship between flow and impact distance, we conduct simulation with input density as 0.5 and the length of channel as 80 and the width as 30 . It is clear that the further the traffic sign is, the larger the flow is within a critical distance. Beyond such distance, flow is getting less and less with increase of the distance. No matter whether the width is 5 or 7 , the largest flow occurs under the condition that impact distance is equal to 30 . If we enlarge the width of the exit to 10 , we find the largest flow occurs when impact distance equals 40 . It concludes that the largest flow occurs when impact distance is equal to or a little bit larger than the channel width. See Figure 7.

Figure 8 shows relationship between flow and input density with various channel lengths. The channel width is set as 30 cells and the length is 60 in Figure 9(a) and 100 in Figure 9(b), respectively. Simulation results show that pedestrian flow remains unchanged with variation of channel length.

To study impact of channel width and traffic sign impact distance on the relationship between flow and input density, we conduct simulation with channel length of 80 cells and exit width of 7 cells. Channel width is set as 20 and 40 cells corresponding to Figures 9(a) and 9(b), respectively. Results show that the largest flow occurs on condition that the width of exit is less than half of the channel width and $d$ is not less than the channel width if the input density is high. This conclusion agrees with results we have got in Figure 7.

To further study the impact of channel size on flow characteristics, we conduct simulation experiments with different channel sizes. 


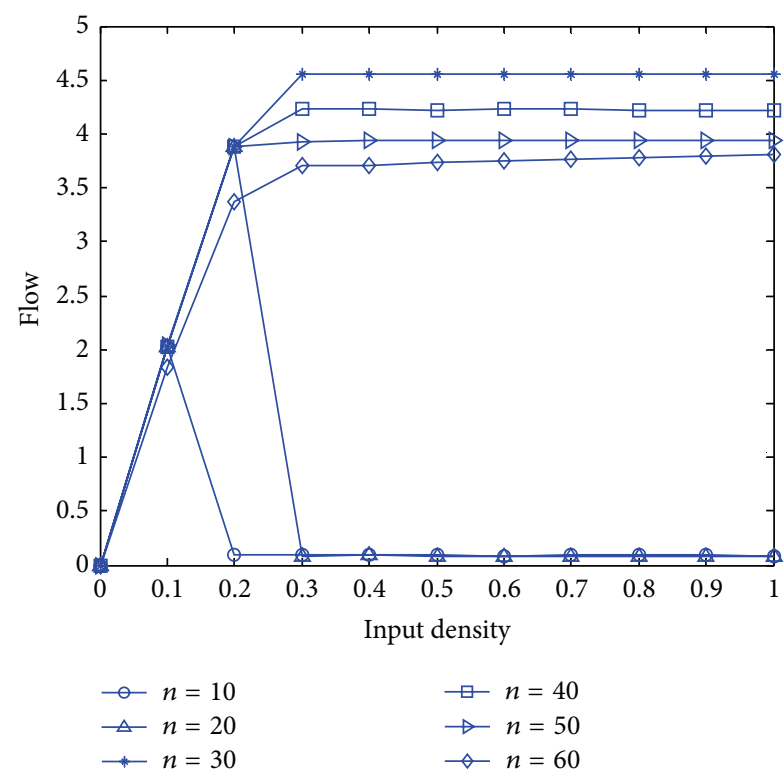

(a)

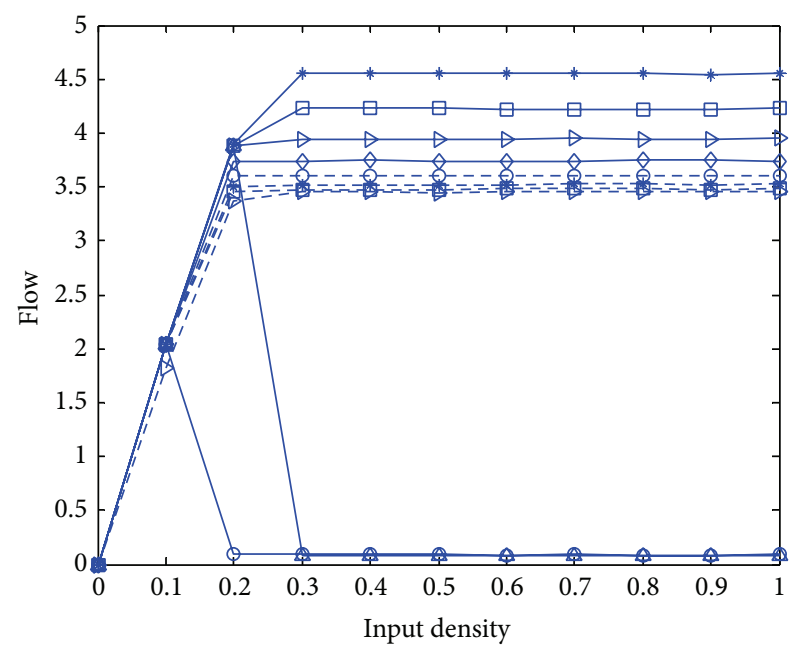

$\rightarrow n=10$
$\triangle n=20$
$\rightarrow-n=30$
$\square n=40$
$\rightarrow n=50$

$\diamond n=60$

$-\theta-n=70$

- *- $n=80$

$-\because-n=90$

$\rightarrow-n=100$

(b)

FIGURE 8: Relationship between flow and input density with various channel lengths. The width of channel is 30 . The length of the channel is set as 60 and 100 cells corresponding to (a) and (b), respectively. See Figure 6(b) for the case with channel length of 80.

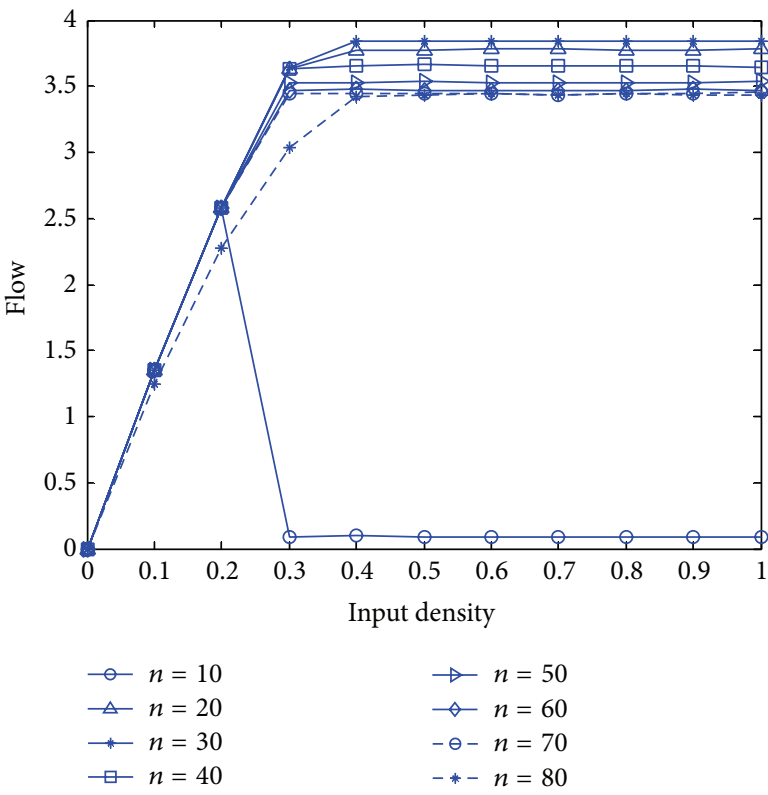

(a)

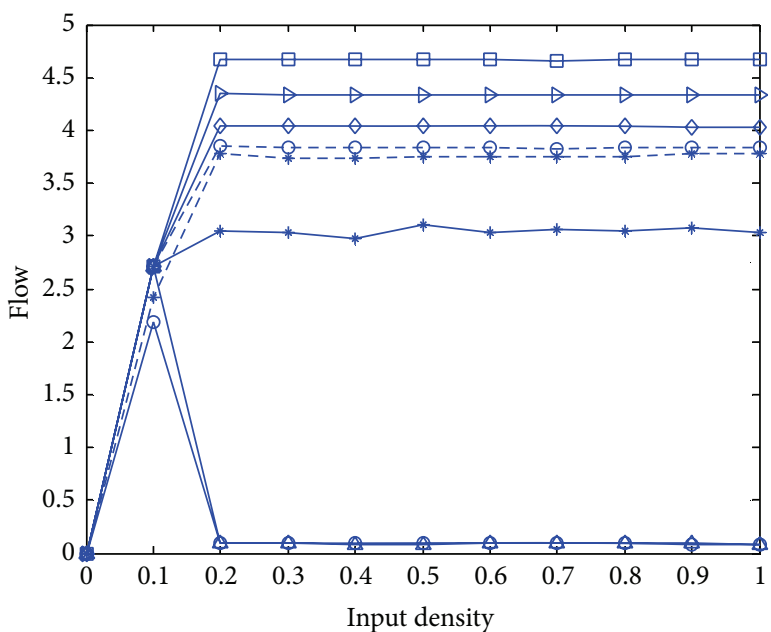

$\rightarrow n=10$
$\triangle n=20$
$\rightarrow-n=30$
$\square n=40$

$\rightarrow n=50$

$\rightarrow n=60$

$-\theta-n=70$

$-*-n=80$

(b)

FIGURE 9: Relationship between flow and input density with different impact distance. The length of channel is 80 . The width of the channel is set as 20 and 40 cells corresponding to (a) and (b), respectively. See Figure 6(b) for the case with channel width of 30.

Setting the width of the channel as 40 cells and the width of each exit as 10 cells, we notice that flow fluctuates when impact distance is 40 cells far from the boundary. See Figure 10. It demonstrates that a critical distance for traffic sign exists. At this distance, pedestrian flow will fluctuate, and, below which, the flow will drop sharply with the increase of input density. With the same input density, the largest flow can only occur on condition that $\mathrm{d}$ is a little bit further than the channel width.

Figures 11(a) and 11(b) are two states in such condition we set above. In Figure 11(a), pedestrians can recognize the exits and move freely to the forward direction, whereas, in 


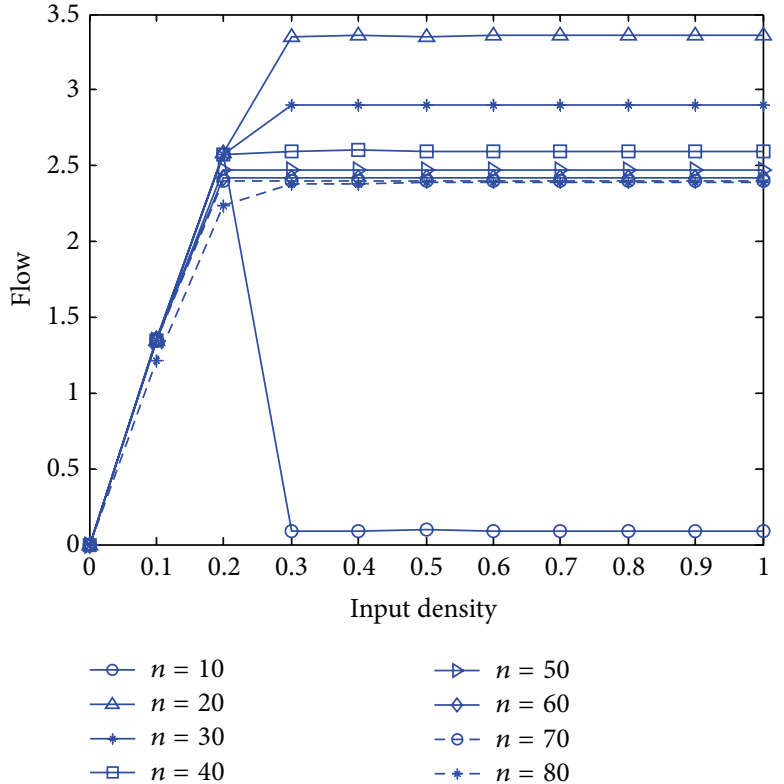

(a)

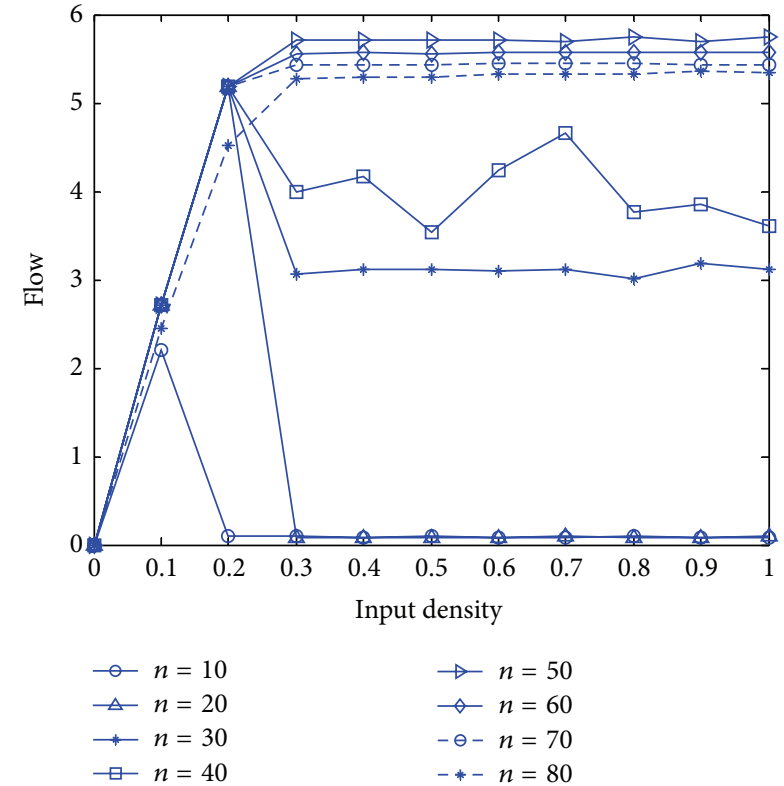

(b)

FIGURE 10: Relationship between flow and input density with different widths of channel and exit. The channel length is 80 . The widths of the channel and exit are set as 20 and 5, and 40 and 10 cells corresponding to (a) and (b), respectively. See Figure 6(b) for the case with channel width of 30 and exit width of 7.

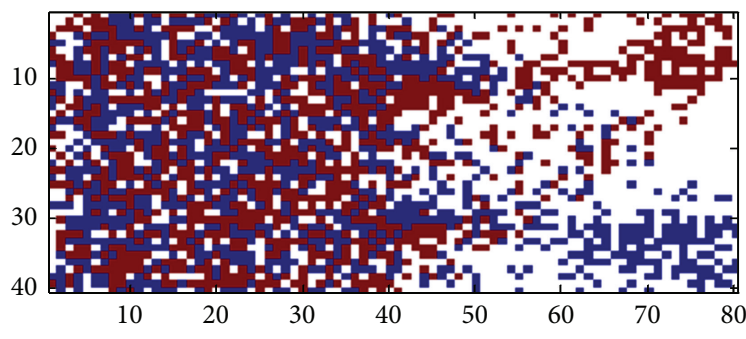

(a)

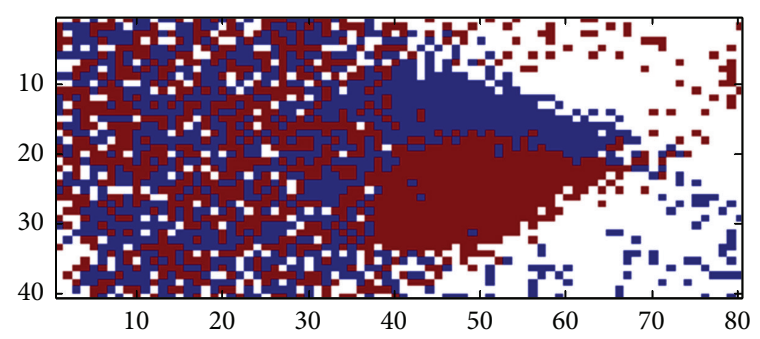

(b)

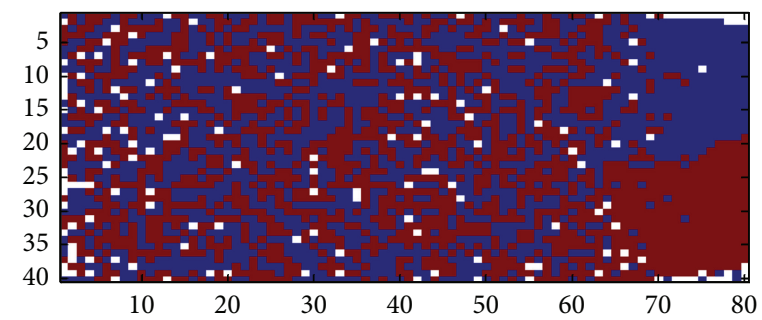

(c)

Figure 11: Pedestrians' moving state. We set channel length as 80 cells, channel width as 40 cells, width of exit as 10 cells, and input density as 0.5 . Two states occurred under this condition showing in (a) and (b). (c) is shown as comparison without traffic sign.

Figure 11(b), there is a block area between pedestrians turning left and those turning right. In Figure 11(c), dead block occurs if the input density is higher than 0.2 without traffic sign.

\section{Conclusion}

In this paper, the impact of traffic sign on pedestrian walking behavior through a channel with two exits has been studied.
The pedestrians are modeled by a cellular automaton model. Two types of pedestrians are considered according to which exit they intend to go through.

We conduct simulation experiments in various scenarios with different channel length, channel width, width of exit, input density, and impact distance. Simulation results indicate that a critical distance of the traffic sign to exit exists for a given channel layout. At the critical distance, pedestrian 
flow fluctuates. Below such critical distance, flow is getting larger with the increase of input density. However, the flow drops sharply if the input density is over a critical level. If the distance is a little bit further than the critical distance, the largest flow occurs and the flow can remain steady no matter what input density will be. Under some conditions in terms of the channel layout, position of traffic sign, and input density, block area between pedestrians turning left and right exists. Without traffic sign, the block area turns into a dead block with some higher input density.

\section{Conflict of Interests}

The authors declare that there is no conflict of interests regarding the publication of this paper.

\section{Acknowledgments}

The authors acknowledge the support from Beijing Municipal Natural Science Foundation with Grant no. 8092026. The authors are grateful to reviewers for the invaluable comments.

\section{References}

[1] R. L. Hughes, "A continuum theory for the flow of pedestrians," Transportation Research Part B: Methodological, vol. 36, no. 6, pp. 507-535, 2002.

[2] M. Di Francesco, P. A. Markowich, J.-F. Pietschmann, and M.-T. Wolfram, "On the Hughes' model for pedestrian flow: the onedimensional case," Journal of Differential Equations, vol. 250, no. 3, pp. 1334-1362, 2011.

[3] D. Amadori and M. Di Francesco, "The one-dimensional Hughes model for pedestrian flow: Riemann-type solutions," Acta Mathematica Scientia B: English Edition, vol. 32, no. 1, pp. 259-280, 2012.

[4] R. M. Colombo and M. D. Rosini, "Pedestrian flows and nonclassical shocks," Mathematical Methods in the Applied Sciences, vol. 28, no. 13, pp. 1553-1567, 2005.

[5] Y. Q. Jiang, S. C. Wong, P. Zhang, R. Liu, Y. Duan, and K. Choi, "Numerical simulation of a continuum model for bi-directional pedestrian flow," Applied Mathematics and Computation, vol. 218, no. 10, pp. 6135-6143, 2012.

[6] Y. Q. Jiang, P. Zhang, S. C. Wong, and R.-X. Liu, "A higher-order macroscopic model for pedestrian flows," Physica A: Statistical Mechanics and its Applications, vol. 389, no. 21, pp. 4623-4635, 2010.

[7] L. F. Henderson, "On the fluid mechanics of human crowd motion," Transportation Research, vol. 8, no. 6, pp. 509-515, 1974.

[8] D. Helbing, "A fluid-dynamic model for the movement of pedestrians," Complex Systems, vol. 6, no. 5, pp. 391-415, 1992.

[9] T. Nagatani, "Freezing transition in bi-directional CA model for facing pedestrian traffic," Physics Letters A: General, Atomic and Solid State Physics, vol. 373, no. 33, pp. 2917-2921, 2009.

[10] V. J. Blue and J. L. Adler, "Cellular automata microsimulation for modeling bi-directional pedestrian walkways," Transportation Research Part B: Methodological, vol. 35, no. 3, pp. 293-312, 2001.

[11] H. Xiong, X. Guo, W. Wang, H. Tan, and H. Wei, "Cellular automaton simulation of counter flow with paired pedestrians,"
International Journal of Computational Intelligence Systems, vol. 4, no. 6, pp. 1415-1421, 2011.

[12] W. F. Fang, L. Z. Yang, and W. C. Fan, "Simulation of bi-direction pedestrian movement using a cellular automata model," Physica A: Statistical Mechanics and its Applications, vol. 321, no. 3-4, pp. 633-640, 2003.

[13] L. Yang, J. Li, and S. Liu, "Simulation of pedestrian counter-flow with right-moving preference," Physica A: Statistical Mechanics and its Applications, vol. 387, no. 13, pp. 3281-3289, 2008.

[14] W. G. Weng, T. Chen, H. Y. Yuan, and W. C. Fan, "Cellular automaton simulation of pedestrian counter flow with different walk velocities," Physical Review E: Statistical, Nonlinear, and Soft Matter Physics, vol. 74, no. 3, Article ID 036102, 2006.

[15] Y. F. Yu and W. G. Song, "Cellular automaton simulation of pedestrian counter flow considering the surrounding environment," Physical Review E: Statistical, Nonlinear, and Soft Matter Physics, vol. 75, no. 4, Article ID 046112, 2007.

[16] Z. Wang, B. Song, Y. Qin, and L. Jia, "Team-moving effect in bidirection pedestrian flow," Physica A: Statistical Mechanics and its Applications, vol. 391, no. 11, pp. 3119-3128, 2012.

[17] M. Muramatsu, T. Irie, and T. Nagatani, "Jamming transition in pedestrian counter flow," Physica A: Statistical Mechanics and its Applications, vol. 267, no. 3, pp. 487-498, 1999.

[18] M. Muramatsu and T. Nagatani, "Jamming transition of pedestrian traffic at a crossing with open boundaries," Physica A: Statistical Mechanics and its Applications, vol. 286, no. 1, pp. 377390, 2000.

[19] Y. Tajima, K. Takimoto, and T. Nagatani, "Scaling of pedestrian channel flow with a bottleneck," Physica A: Statistical Mechanics and its Applications, vol. 294, no. 1-2, pp. 257-268, 2001.

[20] Y. Tajima and T. Nagatani, "Clogging transition of pedestrian flow in T-shaped channel," Physica A: Statistical Mechanics and its Applications, vol. 303, no. 1-2, pp. 239-250, 2002.

[21] R. Jiang and Q. S. Wu, "The moving behavior of a large object in the crowds in a narrow channel," Physica A: Statistical Mechanics and its Applications, vol. 364, pp. 457-463, 2006.

[22] D. Helbing and P. Molnár, "Social force model for pedestrian dynamics," Physical Review E, vol. 51, no. 5, pp. 4282-4286, 1995.

[23] W. J. Yu, R. Chen, L. Y. Dong, and S. Q. Dai, "Centrifugal force model for pedestrian dynamics," Physical Review E: Statistical, Nonlinear, and Soft Matter Physics, vol. 72, no. 2, Article ID 026112, 2005.

[24] M. Chraibi, A. Seyfried, and A. Schadschneider, "Generalized centrifugal-force model for pedestrian dynamics," Physical Review E: Statistical, Nonlinear, and Soft Matter Physics, vol. 82, no. 4, Article ID 046111, 2010.

[25] D. Yanagisawa and K. Nishinari, "Mean-field theory for pedestrian outflow through an exit," Physical Review E: Statistical, Nonlinear, and Soft Matter Physics, vol. 76, no. 6, Article ID 061117, 2007.

[26] H. J. Huang and R. Y. Guo, "Static floor field and exit choice for pedestrian evacuation in rooms with internal obstacles and multiple exits," Physical Review E: Statistical, Nonlinear, and Soft Matter Physics, vol. 78, no. 2, Article ID 021131, 6 pages, 2008.

[27] R. Y. Guo and T. Q. Tang, "A simulation model for pedestrian flow through walkways with corners," Simulation Modelling Practice and Theory, vol. 21, no. 1, pp. 103-113, 2012.

[28] C. Burstedde, K. Klauck, A. Schadschneider, and J. Zittartz, "Simulation of pedestrian dynamics using a two-dimensional cellular automaton," Physica A: Statistical Mechanics and its Applications, vol. 295, no. 3-4, pp. 507-525, 2001. 
[29] D. Helbing, I. Farkas, and T. Vicsek, "Simulating dynamical features of escape panic," Nature, vol. 407, no. 6803, pp. 487490, 2000.

[30] G. Antonini, M. Bierlaire, and M. Weber, "Discrete choice models of pedestrian walking behavior," Transportation Research Part B: Methodological, vol. 40, no. 8, pp. 667-687, 2006.

[31] M. Asano, T. Iryo, and M. Kuwahara, "Microscopic pedestrian simulation model combined with a tactical model for route choice behaviour," Transportation Research C: Emerging Technologies, vol. 18, no. 6, pp. 842-855, 2010.

[32] A. Borrmann, A. Kneidl, G. Köster, S. Ruzika, and M. Thiemann, "Bidirectional coupling of macroscopic and microscopic pedestrian evacuation models," Safety Science, vol. 50, no. 8, pp. 1695-1703, 2012.

[33] J. S. Choi and Y. Morita, "The distribution of signs and pedestrians' walking behaviors in underground space-a case study of the underground shopping center in Taegon, Korea," Journal of Physiological Anthropology and Applied Human Science, vol. 24, no. 1, pp. 117-121, 2005.

[34] M. Liu, X. Zheng, and Y. Cheng, "Determining the effective distance of emergency evacuation signs," Fire Safety Journal, vol. 46, no. 6, pp. 364-369, 2011.

[35] K. Nassar, "Sign visibility for pedestrians assessed with agentbased simulation," Transportation Research Record, no. 2264, pp. 18-26, 2011.

[36] A. Kirchner, K. Nishinari, and A. Schadschneider, "Friction effects and clogging in a cellular automaton model for pedestrian dynamics," Physical Review E, vol. 67, no. 5, Article ID 056122, 2003.

[37] T. Kretz, G. Mayer, and A. Mühlberger, "Behaviour and perception-based pedestrian evacuation simulation," in Pedestrian and Evacuation Dynamics, pp. 827-831, 2011.

[38] G. M. Schutz, Exactly Solvable Models for Many-Body Systems Far from Equilibrium in Phase Transitions and Critical Phenomena, vol. 19, Academic Press, San Diego, Calif, USA, 2001, edited by C. Domb and J. L. Lebowitz.

[39] R. A. Blythe and M. R. Evans, "Nonequilibrium steady states of matrix-product form: a solver's guide," Journal of Physics A: Mathematical and Theoretical, vol. 40, no. 46, pp. R333-R441, 2007.

[40] B. Derrida, "Non-equilibrium steady states: fluctuations and large deviations of the density and of the current," Journal of Statistical Mechanics: Theory and Experiment, no. 7, Article ID P07023, 2007.

[41] A. Schadschneider, D. Chowdhury, and K. Nishinari, Stochastic Transport in Complex Systems: From Molecules to Vehicles, Elsevier, New York, NY, USA, 2010. 


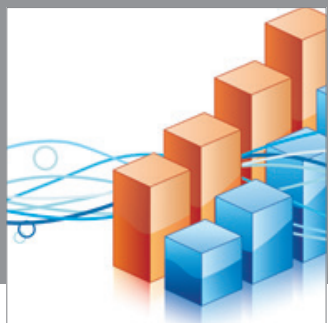

Advances in

Operations Research

mansans

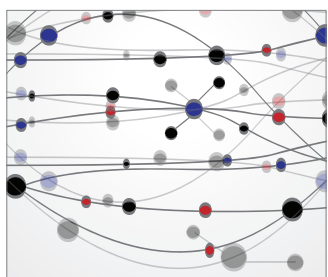

The Scientific World Journal
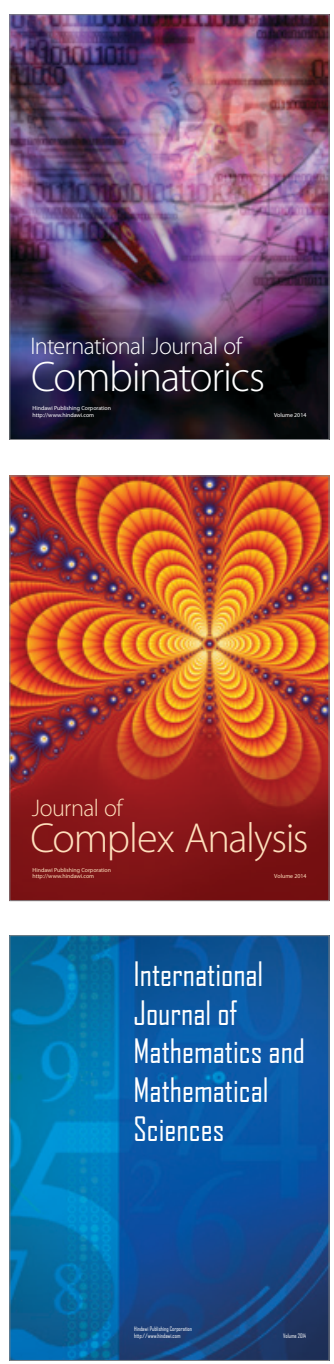
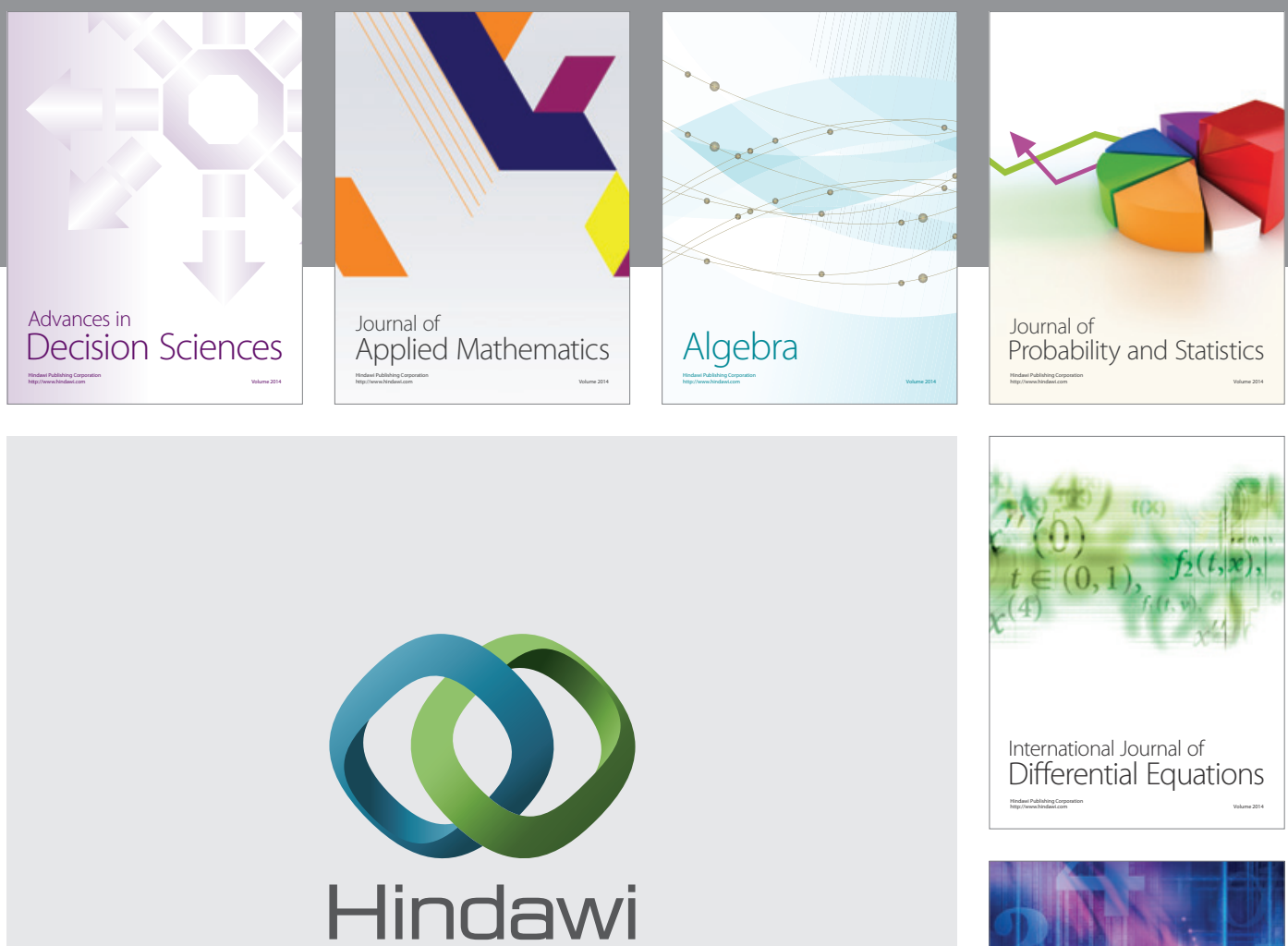

Submit your manuscripts at http://www.hindawi.com
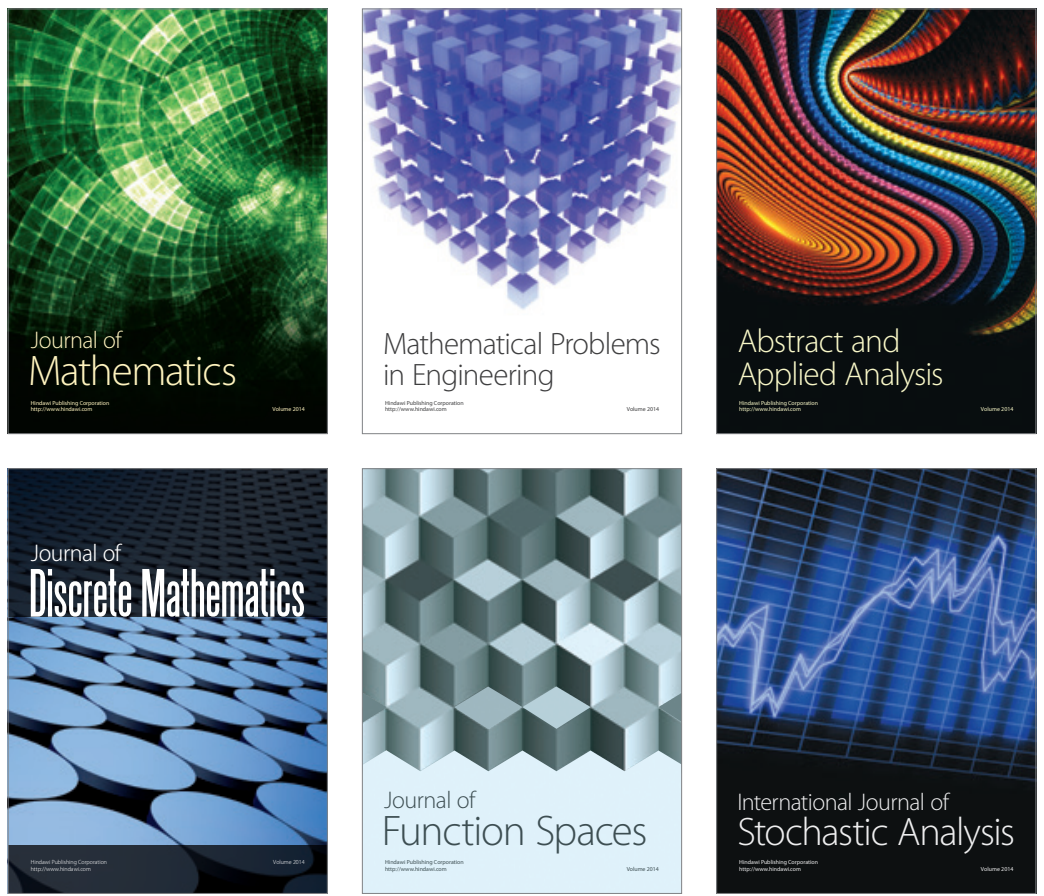

Journal of

Function Spaces

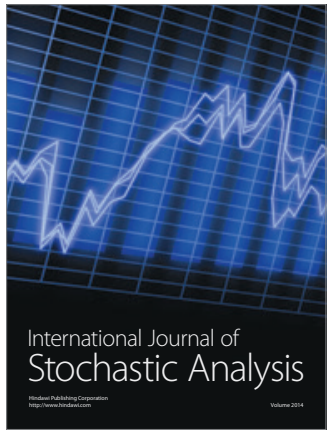

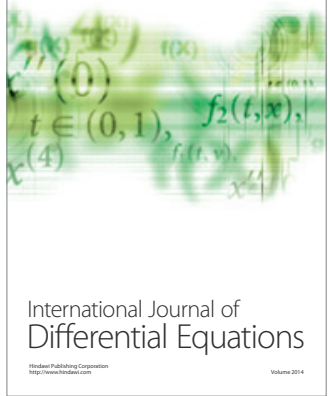
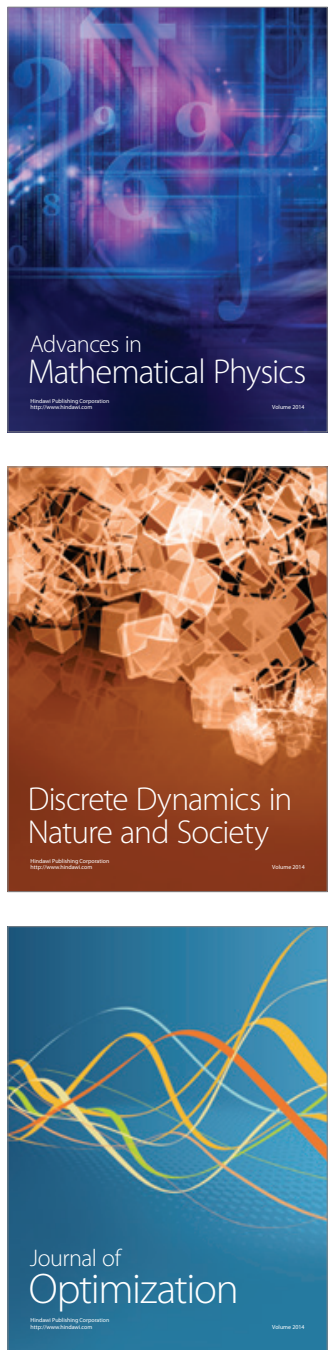